ACCEPTED MANUSCRIPT

\title{
A mechanical system for tensile testing of supported films at the nanoscale
}

To cite this article before publication: Maria F Pantano et al 2018 Nanotechnology in press https://doi.org/10.1088/1361-6528/aacf50

\section{Manuscript version: Accepted Manuscript}

Accepted Manuscript is "the version of the article accepted for publication including all changes made as a result of the peer review process, and which may also include the addition to the article by IOP Publishing of a header, an article ID, a cover sheet and/or an 'Accepted Manuscript' watermark, but excluding any other editing, typesetting or other changes made by IOP Publishing and/or its licensors"

This Accepted Manuscript is @ 2018 IOP Publishing Ltd.

During the embargo period (the 12 month period from the publication of the Version of Record of this article), the Accepted Manuscript is fully protected by copyright and cannot be reused or reposted elsewhere.

As the Version of Record of this article is going to be / has been published on a subscription basis, this Accepted Manuscript is available for reuse under a CC BY-NC-ND 3.0 licence after the 12 month embargo period.

After the embargo period, everyone is permitted to use copy and redistribute this article for non-commercial purposes only, provided that they adhere to all the terms of the licence https://creativecommons.org/licences/by-nc-nd/3.0

Although reasonable endeavours have been taken to obtain all necessary permissions from third parties to include their copyrighted content within this article, their full citation and copyright line may not be present in this Accepted Manuscript version. Before using any content from this article, please refer to the Version of Record on IOPscience once published for full citation and copyright details, as permissions will likely be required. All third party content is fully copyright protected, unless specifically stated otherwise in the figure caption in the Version of Record.

View the article online for updates and enhancements. 


\section{A mechanical system for tensile testing of supported films at the nanoscale}

\section{Maria F. Pantano ${ }^{1}$, Giorgio Speranza $a^{2,3}$, Costas Galiotis ${ }^{4,5}$, Nicola Pugno ${ }^{1,6,7^{*}}$}

${ }^{1}$ Laboratory of Bio-inspired \& Graphene Nanomechanics, Department of Civil, Environmental and Mechanical Engineering, University of Trento, Via Mesiano 77, 38123 Trento, Italy

${ }^{2}$ Center for Materials and Microsystems, Fondazione Bruno Kessler, Via Sommarive 18, 38123 Povo (TN), Italy

${ }^{3}$ Department of Industrial Engineering, University of Trento, via Sommarive 9,38123 Trento, Italy

${ }^{4}$ Department of Chemical Engineering, University of Patras, Rio Patras, 26504, Greece

${ }^{5}$ Institute of Chemical Engineering Sciences, Foundation of Research and Technology-Hellas (FORTH/ICE-HT), Stadiou Street, Platani, Patras Acahaias, 26504, Greece

${ }^{6}$ School of Engineering and Materials Science, Queen Mary University of London, Mile End Road, London E1 4NS, U.K.

${ }^{7}$ Ket-Lab, Edoardo Amaldi Foundation, Italian Space Agency, Via del Politecnico snc, 00133 Rome, Italy

*Corresponding author: nicola.pugno@unitn.it; Tel.: +390461282525.

Abstract. Standard tensile tests of materials are usually performed on freestanding specimens. However, such requirement is difficult to implement when the materials of interest are of nanoscopic dimensions due to problems related to their handling and manipulation. In the present paper, a new device is presented for tensile testing of thin nanomaterials, which allows tests to be carried out on specimens initially deposited onto a macroscopic pre-notched substrate. On loading, however, no substrate effects are introduced, allowing the films to be freely stretched. The results obtained from a variety of thin metal or polymeric films are very promising for the further development of this technique as a standard method for nanomaterial mechanical testing.

Keywords: Nanomaterials, thin films, tension, mechanical properties

\section{Introduction}

Nanoscale materials, like nanowires, nanotubes and nanofilms, display fascinating properties [1-2] that can be exploited in a plethora of possible applications, including composites [3-4], electronic devices [5-6], flexible electronics [7-9], batteries [10] and biosensors [11]. 
In order to fully exploit the great potential of such new materials into the design of high-performance yet reliable devices, it is necessary to deeply understand their mechanical behavior. However, the small size of these materials makes their experimental investigation challenging, as the standardized well-assessed methodologies and equipment usually involved at the macroscale are not effective for manipulation of micro/nanosized components [12].

Thus, new tools have to be developed for assessing the mechanical behavior at the nanoscale. In recent years, very useful systems for tensile testing of micro/nanosamples have been proposed based on Micro-Electro-Mechanical Systems (MEMS) technology [13-21]. These consist of miniaturized testing machines, with the unique advantage of being compatible with electron microscopes, which enable a real time monitoring of the sample deformation.

However, while many of these have been successfully applied to one-dimensional specimens, like nanowires and nanotubes, as well as thin films, the tested volume of the materials is indeed extremely small and the results are not always reliable [22-23].

Another limitation concerning tensile tests on nanomaterials or thin films is related to the manipulation and fabrication of completely freestanding samples, requiring new and proper metrological strategies to be designed. For example, up to date, the only experimental data about Young modulus, fracture strength and strain of single atomic layer materials are still those derived from a couple of nanoindentation and bending tests [24-25], where thin sheets of material are suspended over an array of open holes and loaded with the tip of an Atomic Force Microscope (AFM). In all these cases true uniaxial stress-strain curves cannot be obtained as the testing conditions are in essence biaxial.

In the present paper, we report a new mechanical device, which can be very promising for tensile testing of large-area thin films and can be developed even for testing 2D materials. The tests are required to be carried out on a specimen initially deposited onto a substrate, thus addressing the issue related to availability of completely freestanding samples. A detailed description of its design and calibration methodologies are proposed in the next sections, followed by an application to microwires and thin films with known properties for validation.

\section{Device concept}

One of the main issues related to tensile testing of nanoscale thin films is concerned with the availability and manipulation of freestanding samples. Nowadays, there are well established experimental protocols, as those based on Chemical Vapor Deposition (CVD), which allow to grow relatively large-area samples $\left(>1 \mathrm{~cm}^{2}\right)$ on suitable substrates, like copper or nickel in the case of graphene [26-27]. However, in order to have freestanding samples, these have to be peeled up and 
fixed to a testing machine through effective grips. While there are assessed transfer techniques to move a nanoscale thin film from its native substrate onto an arbitrary structure [28], still remain difficulties into manipulation of completely freestanding sheets.

Similar issues were already faced in the 1990s when films with few micrometers thickness started to be tested. For example, at that time, in order to simplify manipulation and alignment, freestanding samples were fabricated with dog-bone shape and supporting strips [29-30] or cofabricated within the whole testing machine by lithography [31]. However, in the case of 2D materials, there are many technical issues, which limit availability of completely freestanding samples with non-rectangular shape. Thus, alternative strategies have to be followed, with some examples involving a specimen supported by non-conventional substrates, like compliant substrates or water films [32-33]. However, in the first case, careful data processing is required in order to isolate the substrate influence, which is believed to increase as the sample thickness decreases. The second strategy, which proposes samples supported by a water film, is only valid when samples are not reactive with the supporting liquid and van der Waals forces, used for gripping to the loading frame, are sufficient to avoid sliding (i.e., in the case of ultra-strong materials other gripping mechanisms could be necessary).

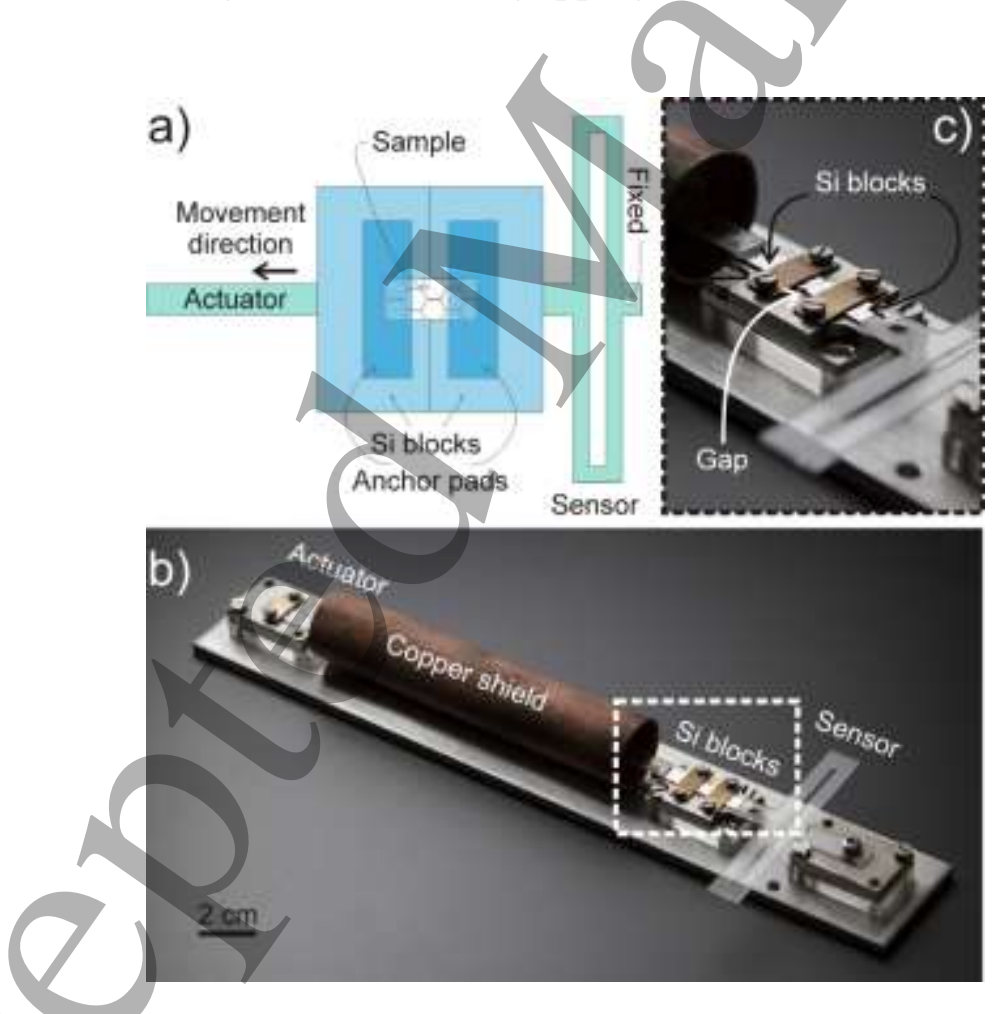

Figure 1. (a) Design of the device for tensile testing of nanoscale thin films, consisting of a thermal actuator, a flexible structure with load sensing function (i.e., sensor), and two faced Si blocks supporting the sample to be tested (not scaled). While testing (b), the actuator is covered with a copper shield to guarantee homogeneous heat distribution over the actuating beam. The actuating beam is glued to one Si block separated by a few micrometer gap from another Si block, which is attached to 
the sensor, as shown in the zoomed view (c). The metallic clips spanning over the Si blocks are used for alignment purposes, and they are removed during sample preparation, thus before starting the test.

In the design of our system, we were able to combine all the advantages guaranteed by deposited samples (i.e., ease of deposition and manipulation) to the availability at the end of a freestanding sample, whose behavior is thus not affected by the substrate. In fact, in our case the specimen is initially deposited onto a pre-notched substrate, which is fractured in two facing blocks separated by a small gap in between $(<3 \mu \mathrm{m})$. This narrow fracture gap ensures the specimen is not bent. Each side of the substrate is glued to either an actuator, in order to apply load/displacement, or a sensor, which has the task to measure the load/displacement delivered to the sample (figure 1).

In our experiments, the sample supporting frame was made of silicon, since this is provided with crystalline planes, where fracture, once initiated, proceeds in a very sharp mode, providing two facing plates with a very small and well defined gap in between, as required by our design.

Then, as soon as the actuator is activated, the Si block attached to it moves apart from the other, thus stretching the sample. This in turn transfers part of the delivered displacement to the sensor.

Because of its macroscopic size, the stage is relatively easy to manipulate and can accommodate large-area samples with characteristic length in the order of $100 \mu \mathrm{m}-1 \mathrm{~mm}$. Furthermore, the system is compatible with observation under optical microscope, which guarantees tests to be followed in real-time.

\section{Device design and calibration}

The device developed herein is purely mechanical, i.e., it does not require any complex electronics to operate. In fact, the actuator is based on a thermal working principle, consisting of a Poly(vinyl chloride) (PVC) beam, which deforms as a consequence of temperature increase. In particular, after the sample is positioned on the device stage and its substrate is broken in two facing blocks according to a procedure explained later in more details, the actuator is heated by light bulbs. As a consequence, it deforms in the direction opposite to the sample. Then, heating is turned off and the actuator end that was free to expand up to that time is fixed through a glue drop. Thus, the actuator starts to contract, pulling the sample.

The sensor is a flexible structure, which behaves as a spring connected in series to the sample. As the specimen is pulled by the actuator, the sensor undergoes a displacement proportional to the force on the specimen. Thus, the sensor deformation can be derived from processing a series of images taken during the test through an optical microscope, and this can be multiplied by the sensor spring constant, in order to obtain the corresponding load. 
From a fabrication point of view, the actuator requires a material characterized by high thermal expansion coefficient and lightweight properties, since it consists of a long freestanding beam, which should not bend significantly under its own weight. From the sensor point of view, a lightweight material is required, as well, but in addition it should be provided with medium-low mechanical properties, in order to design a spring with sufficiently low stiffness and macroscopic size, thus guaranteeing high load resolution and ease of manipulation.

All these requirements were successfully addressed with the choice of PVC as structural material, whose main mechanical and physical properties are collected in Table 1. Films with $1 \mathrm{~mm}$ thickness were then considered for the design of both the sensing and actuating parts.

The design of the actuating beam was performed on the base of two main considerations. First, this has to be able to deliver high displacements (in the order of $100 \mu \mathrm{m}$ ) at a relatively low temperature increase $\left(\sim 10{ }^{\circ} \mathrm{C}\right)$ in order to avoid a significant temperature increase also in the sample, which in turn can affect its mechanical behavior. Second, the actuator should have a sufficiently higher stiffness than that of the sample in order for its displacement not to be affected when a specimen is mounted. The displacement produced by thermal expansion of the actuating beam in its nearly free condition can be computed as:

$\Delta l=\alpha \cdot \Delta T \cdot l_{0}$

where $\alpha$ is the PVC thermal expansion coefficient (Table 1), $\Delta T$ is the desired temperature increase, and $l_{0}$ is the initial beam length. According to eq. (1), an initial length of $15 \mathrm{~cm}$ can accommodate elongation bigger than $100 \mu \mathrm{m}$ at $\Delta T=10^{\circ} \mathrm{C}$. During our experiments, the actuator is typically heated for less than 3 minutes, which result in a temperature increase at the sample location not exceeding $3^{\circ} \mathrm{C}$.

Table 1. Physical and mechanical properties of PVC.

$\begin{array}{lc}\text { Young Modulus, } E[\mathrm{GPa}] & 3.2 \\ \text { Thermal expansion coefficient, } \alpha\left[\cdot 10^{-5}{ }^{\circ} \mathrm{C}^{-1}\right] & 5 \\ \text { Density, } \rho\left[\mathrm{kg} / \mathrm{m}^{3}\right] & 1400\end{array}$

In order to provide sufficient stiffness $(>200 \mathrm{kN} / \mathrm{m})$, the actuator beam was designed with $5 \mathrm{~mm}$ width. 
The sensor C-shaped geometry was borrowed from typical spring configurations used in MEMSbased devices. Its stiffness can be computed as the flexural stiffness of a beam clamped at one end and guided at the other end:

$k_{s}=k=\frac{E b^{3} t}{l^{3}}$

where $k$ is the stiffness of one branch (i.e., beam), $E$ is the PVC Young modulus (Table 1), $b$ and $l$ are the width and the length of each beam, respectively, and $t$ is the thickness $(t=1 \mathrm{~mm})$. We chose $l=26$ $\mathrm{mm}, b=4 \mathrm{~mm}$, thus providing $k=10900 \mathrm{~N} / \mathrm{m}$.

After fabrication, the sensor was calibrated by hanging weights of known mass. As in [34], the sensor was fixed vertically to the base of an optical microscope, in order to monitor the deflection caused by application of mass samples hang through a hook (figure 2). To simplify the measurement, such deflection was evaluated as the displacement of a microwire attached to the sensor with respect to a reference microwire, kept fixed during the test. As expected, a linear relationship resulted between force and displacement, with the calibration constant being $7500 \pm 120 \mathrm{~N} / \mathrm{m}$, which is smaller than the design value, but in good accordance with $7426 \mathrm{~N} / \mathrm{m}$, which is the value obtained by numerical simulation of the sensor. Such difference between the result provided by eq. (2) and the experimental/numerical value is due to the nature of PVC, which is too soft to reproduce the ideal constraints for analytical model (2) to be valid.
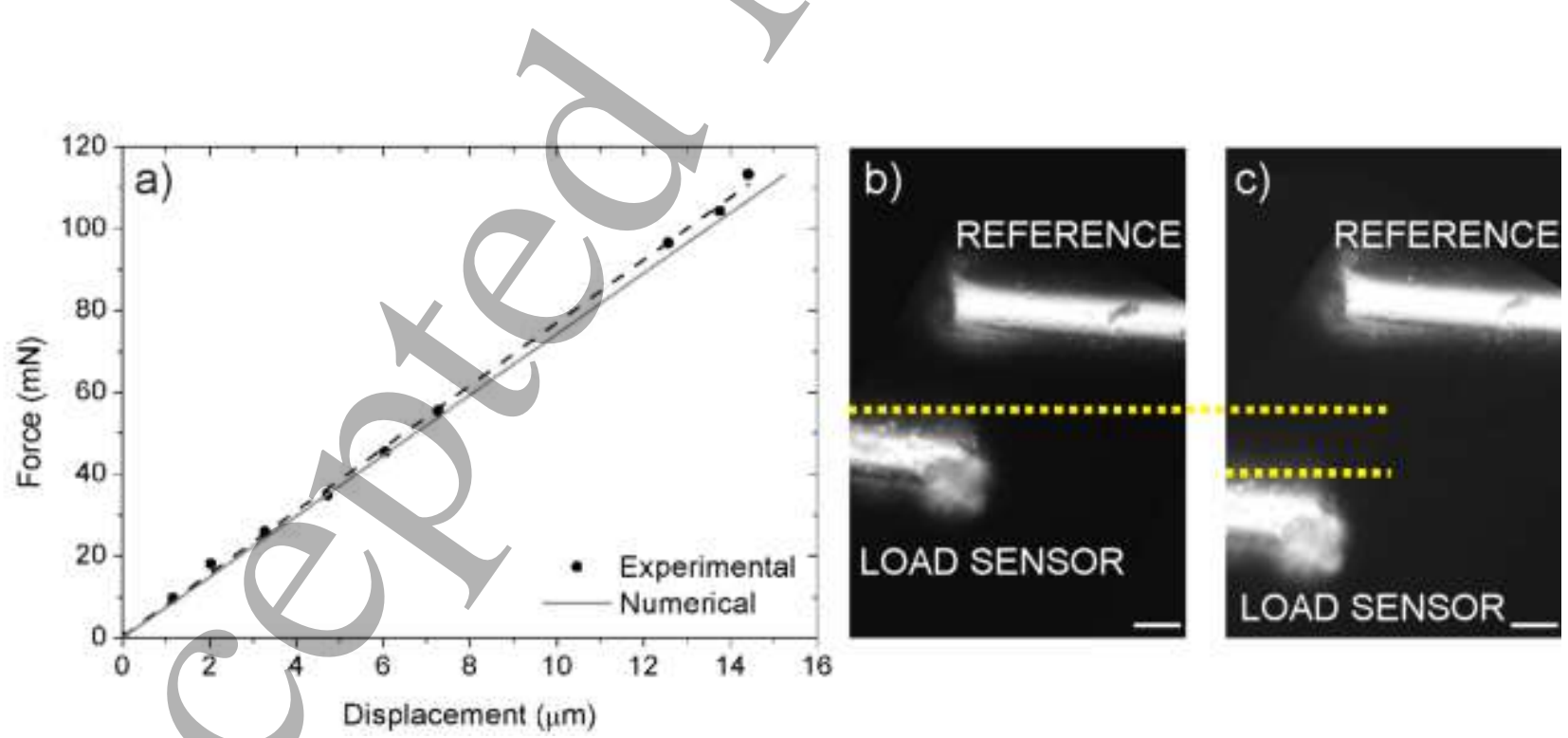

Figure 2. (a) Comparison between the sensor calibration curve obtained from experiments (dotted line) and from numerical simulations (solid line). The sensor displacement caused by hanging reference masses (i.e., reference forces) was evaluated measuring the displacement of a microwire attached to it with respect to a reference under an optical microscope. Position of the load sensor 
microwire with respect to the reference at rest (b) and after applying $100 \mathrm{mN}$ force (c). Scale bar: 10 $\mu \mathrm{m}$.

The calibration test was repeated three times with good repeatability within the force range $0-0.1 \mathrm{~N}$ that is the interval expected to be spun during tests on samples.

\section{Results and Discussion}

Even if the device was designed for testing nanoscale thin films, it is versatile and can be effectively applied to specimens with different geometry, such as 1D and 2D micro/nanostructures, too. Thus, in order to demonstrate the validity of the present design and its suitability to test specimens with both 1D and 2D geometry, aluminum microwires and aluminum nanofilms were tested. The validity of the obtained results, in terms of mechanical properties of the tested specimens, was then assessed through comparison with reference values derived from tests performed on the same specimens through a commercial nanotensile testing machine (Agilent T150 UTM). Finally, the device was applied for testing Poly(methyl methacrylate) (PMMA) films with about $100 \mathrm{~nm}$ thickness.

Before proceeding with the mechanical test and once the $\mathrm{Si}$ substrate with the specimen on top is glued to both the actuator and the load sensor, a sharp tip engraves the Si substrate in order to cause the onset of a fracture line running parallel to its groove on the back. In order to avoid any undesirable movement, which could cause damage to the specimen, the Si substrate is kept firmly in place by a mechanical clamp that presses it against a fixed surface (figure 3). The effectiveness of the mechanical clamp is confirmed by the tiny width of the fracture line (that is limited to few micrometers) and the absence of defects induced in specimens during the substrate fracture. After fracture, the clamp is released and the actuator is activated as described above in order to perform the tensile test with real time observation under an optical microscope. 


\section{a)}

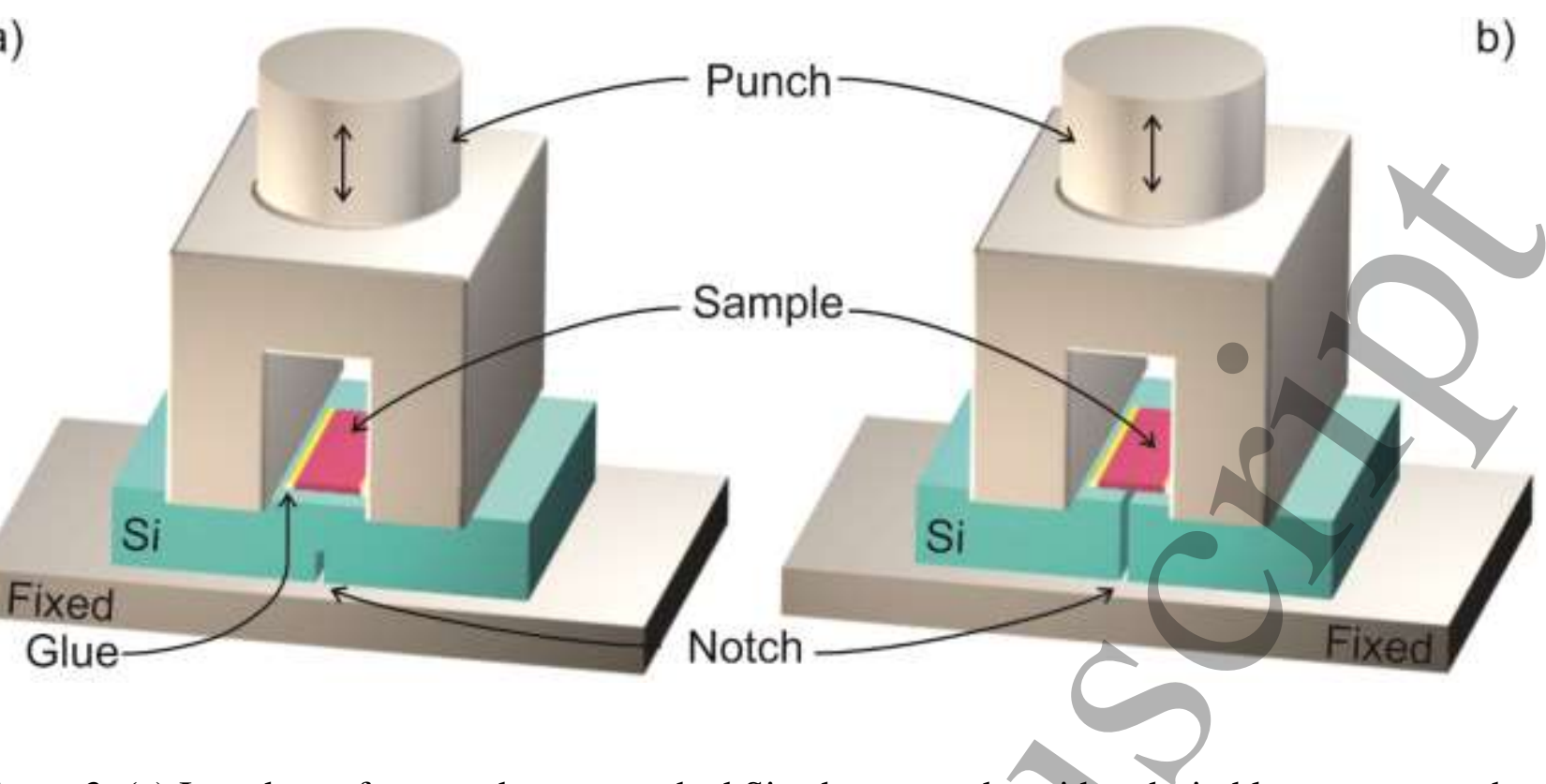

Figure 3: (a) In order to fracture the pre-notched Si substrate and avoid undesirable movements that could induce damage to the specimen positioned on top (and aligned with the Si notch on the back), the Si substrate is pressed by a punch against a fixed surface. At this stage, the actuator and the sensor (not shown in the picture) are already connected to the Si substrate. With the help of a sharp tip, fracture initiates from the pre-notch and propagates, releasing the central part of the specimen (b). Then, the punch moves upwards, releasing the full testing system, which finally moves under the objective of an optical microscope.

Such preliminary steps, including the substrate fracture and fixture to the loading and sensing structures, have to be repeated each time a new specimen has to be tested.

Figure 4 reports the stress-strain curve of an aluminum microwire with a diameter of $18 \mu \mathrm{m}$, which was tested in quasistatic conditions at a strain rate of $1.6 \cdot 10^{-4} \mathrm{~s}^{-1}$. For strain calculation, the initial length was chosen equal to the distance between the glue drops anchoring the specimen to the substrate before this was fractured. The curve is very detailed, being the result of more than 130 data points and captures significant features as localized load drop events in the plastic regime. With reference to the mechanical properties, the Young modulus resulted to be $39 \pm 2 \mathrm{GPa}$, the fracture strength equal to $233 \pm 7 \mathrm{MPa}$ and the fracture strain equal to $4.8 \pm 0.3 \%$. Such values are in good agreement with those derived from a tensile test carried out on the same specimen with a commercial nanotensile testing machine (Agilent T150 UTM), which provided reference values of Young modulus of $45 \mathrm{GPa}$, a fracture strength of $249 \mathrm{MPa}$ and a fracture strain of $3.5 \%$. 

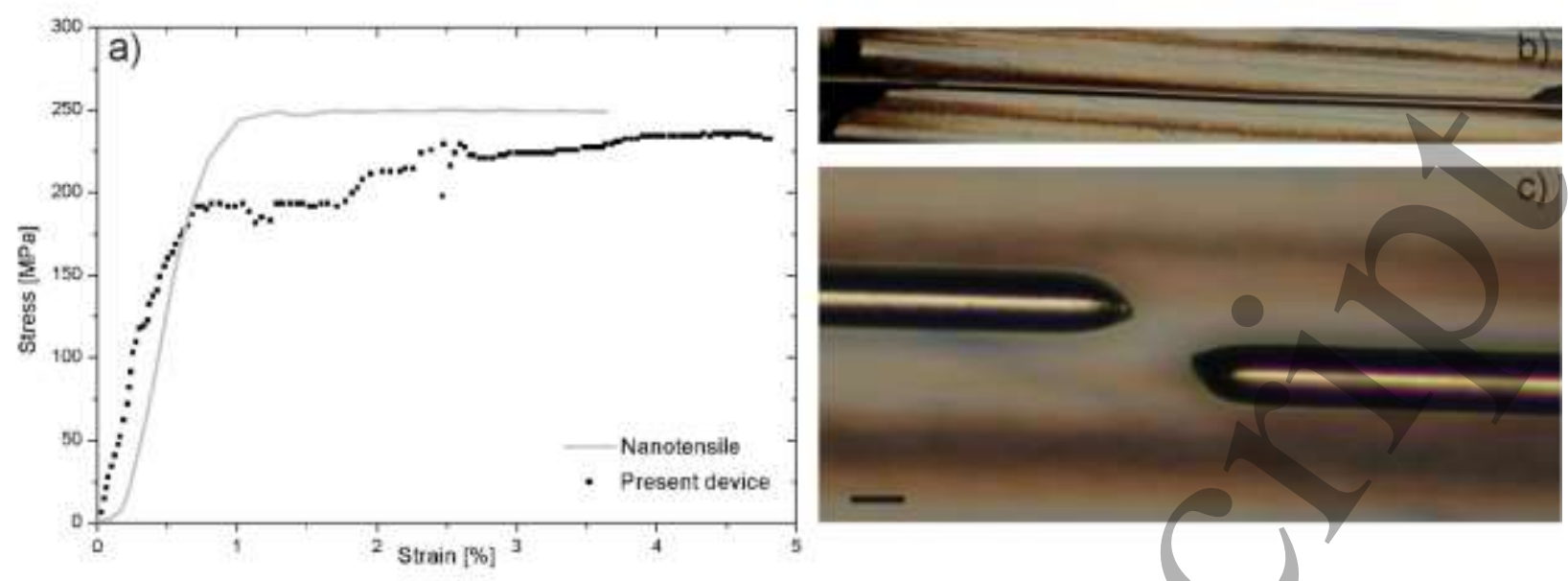

Figure 4. (a) Stress-strain curve of a $18 \mu \mathrm{m}$ aluminum microwire tested with either a commercial nanotensile tester (gray curve) or the present device (black dots). (b) An optical microscope picture of the tested specimen mounted onto the Si substrate before fracture. (c) Optical image of the specimen after failure, showing evidence of necking. Scale bar: $15 \mu \mathrm{m}$.

Figure 5a reports an example stress-strain curve of an aluminum film with $800 \mathrm{~nm}$ thickness, about $750 \mu \mathrm{m}$ length and $1200 \mu \mathrm{m}$ width, which was tested at a strain rate of $4.4 \cdot 10^{-6} \mathrm{~s}^{-1}$. Also in this case, many data points (about 120) were recorded. With respect to the aluminum microwire, a comparable strength was achieved $(257 \pm 2 \mathrm{MPa})$ but at a significantly smaller strain $(\sim 0.14 \pm 0.03 \%)$. However, given their different geometry and application field (our tested microwires are commercial components used for wire bonding applications while nanofilms are commercial light filters), we expect that microwires and nanofilms were produced by a different manufacturing process that could have provided $\mathrm{Al}$ with a different microstructure (i.e., grain size and grain boundary phases). Indeed, it is well known that variations in the fabrication process parameters can have a significant influence on $\mathrm{Al}$ and $\mathrm{Al}$ alloys mechanical properties [35-36]. In order to have some reference values for a comparison, we then conducted tests on similar films with the commercial nanotensile tester (figure 5a). However, this requires specimens to have a macroscopic size (about $2 \times 10 \mathrm{~mm}^{2}$ ), which is challenging to cut without introducing defects. A specimen with $2.5 \mathrm{~mm}$ width and $11 \mathrm{~mm}$ resulted into a fracture strain of $0.5 \%$ and a strength of $23 \mathrm{MPa}$, which is one order of magnitude smaller. However, in the case of our device, the sample had a smaller extension, causing also a smaller defects distribution. As a matter of fact, a test on a specimen with the biggest size compatible with our device, provided a strength of about $50 \mathrm{MPa}$. Thus, the difference between the results obtained with our device and the nanotensile tester can be evidence of defects induced during sample preparation in the 

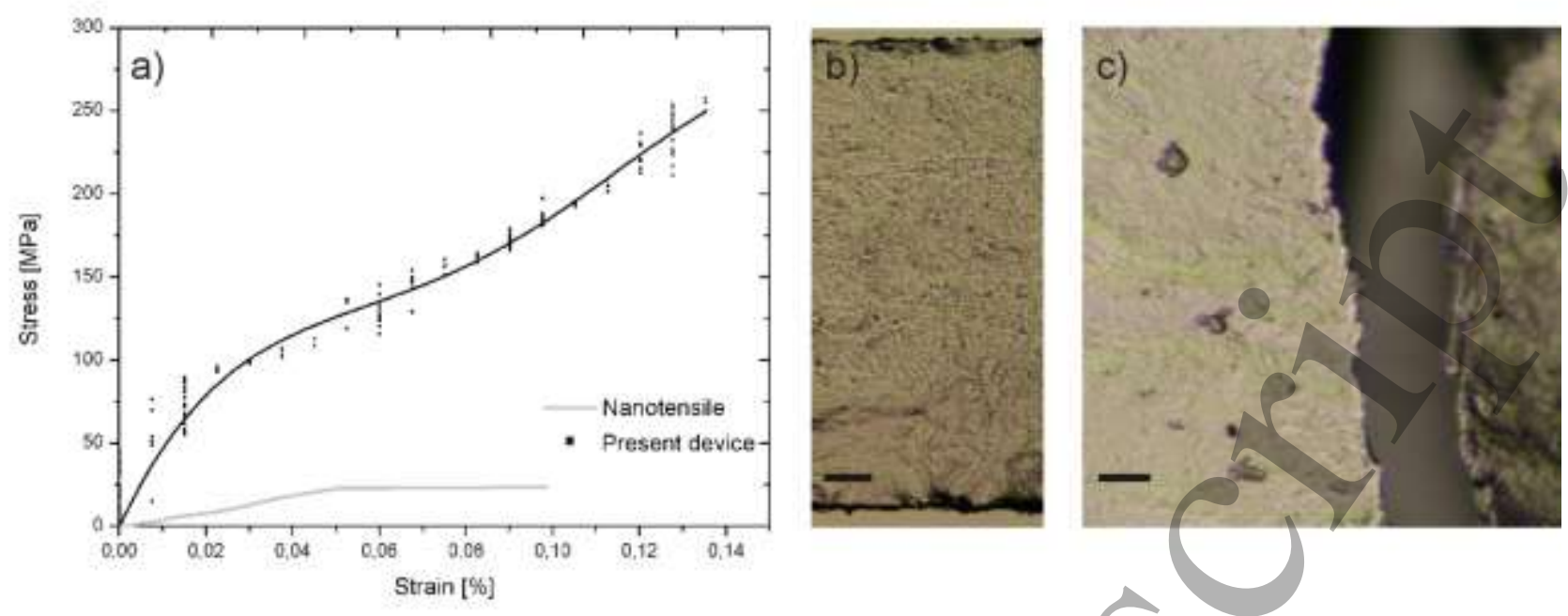

Figure 5. (a) Stress-strain curve of a $800 \mathrm{~nm}$ thick aluminum foil tested with either a commercial nanotensile tester (gray curve) or the present device (black dots). (b) An optical microscope picture of the tested specimen deposited onto the Silicon substrate before fracture. Scale bar: $100 \mu \mathrm{m}$. (c) Optical image of the specimen after failure. Scale bar: $15 \mu \mathrm{m}$.

Finally, the device was successfully applied for the mechanical characterization of PMMA nanofilms. In this case, given the small thickness of the specimen, a slight modification was introduced to the system in order to avoid any substrate effects. In fact, while in the previous cases, there was a thin air cushion between the specimen and the substrate, the strong adhesion of PMMA to Si prevents the possibility to break the substrate without damaging the PMMA film, too. Thus, a substrate with an additional pre-notch on top was used in order to provide a localized freestanding sample region. Furthermore, given the true nanoscale thickness of this film compared to previous material samples, we expect smaller forces to be involved, which suggest to improve the load sensing resolution of our device through the implementation of a new load sensor. Compared to the previous design, the new load sensor has longer $(35 \mathrm{~cm})$ and thinner $(2.5 \mathrm{~cm})$ branches, which provide it with a stiffness of 831 $\mathrm{N} / \mathrm{m}$ (evaluated experimentally as described in Section 3).

Figure 6 reports a stress-strain curve (a) of a PMMA film with $100 \mathrm{~nm}$ thickness, $2.8 \mathrm{~mm}$ width and $123 \mu \mathrm{m}$ gage length and a couple/of optical images of the same sample before and after the tensile test (Figure 6b-c). Again, from the stress-strain curve we can derive the strength, the strain at break and the Young modulus of the sample, that resulted to be $21 \mathrm{MPa}, 18.8 \%$ and $\sim 2.4 \mathrm{GPa}$, respectively. Such values are comparable yet lower than those reported for commercial PMMA particularly regarding the tensile strength values (tensile strength of 40-80 MPa, strain at break of 5-40\% and Young modulus of 1.8-3.3 GPa). However, the data provided by commercial manufacturers refer to bulk properties, which can be significantly different from nanoscale properties as a consequence for 
example of a completely different fabrication process or size effect. Given the role played by PMMA in several industrial applications, including biomedical devices, the investigation of its nanoscale mechanical properties will be the object of a future study.
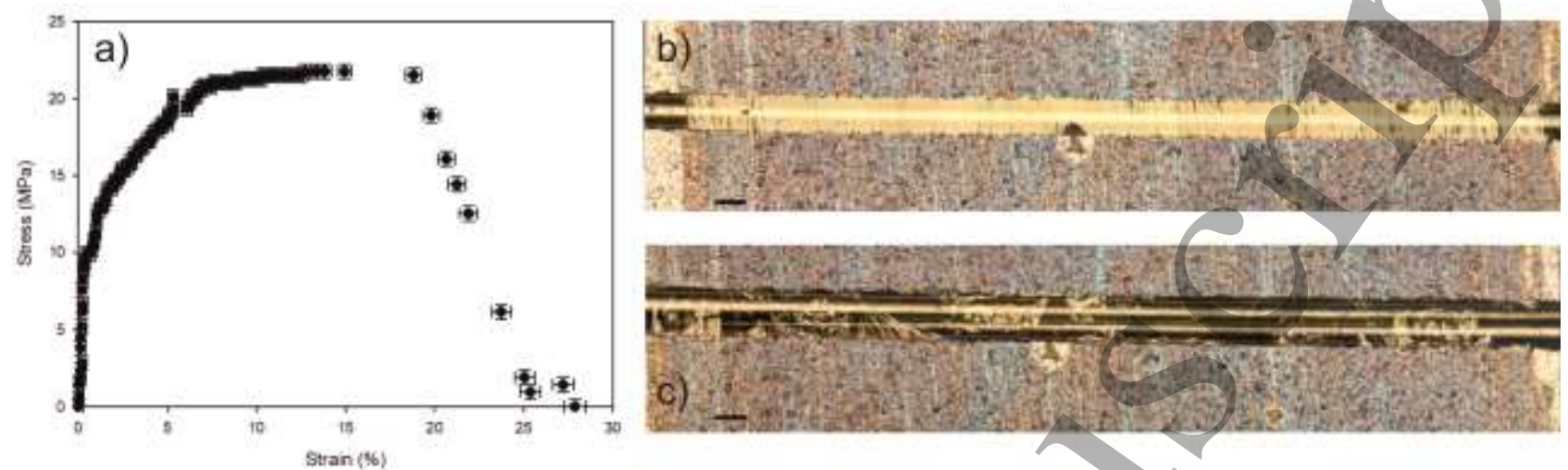

Figure 6. (a) Stress-strain curve of a $100 \mathrm{~nm}$ thick PMMA film tested with the present device. Optical microscope pictures of the tested specimen before (b) and after (c) the tensile test. The specimen was freestanding over a window of about $120 \mu \mathrm{m}$ width corresponding to a groove in the Si substrate (central dark area in the pictures). Scale bar: $100 \mu \mathrm{m}$.

\section{Concluding remarks}

The design and characteristics of the device reported here enable the effective tensile testing of nanomaterial samples. First of all, specimens are initially deposited on two facing blocks separated by a small gap in between, which were obtained in the present configuration from a pre-notched silicon substrate, without the need of completely freestanding samples. Moreover, the system has a macroscopic size, which offers the possibility to work on large-area samples, and does not require any complex electronics, which simplifies significantly its operation protocol. In fact, tests can be performed in air under an optical microscope, with the further advantage to have direct and easy access to the sample, which in turn offers the possibility to perform more than purely mechanical tests. On the other side, operation under high resolution microscopes would allow to capture nanoscale phenomena as fine dislocation and void nucleations. Direct comparison with the results obtained with a nanotensile test on 1D microwires has confirmed the efficiency of the device, whereas that on thin films suggests the limit of conventional tensile testing machines for nanoscale thin films. A wide yariety of materials can be tested with our device, including both metals and polymers, micro and nanoscale specimens. Indeed, our device is versatile and can be efficiently customized according to the sample of interest. For example, in the case of true nanoscale films, such as our tested PMMA nanofilms, the load sensor geometry was slightly modified in order to provide enhanced load 
measurement resolution. Similarly, also the thermal actuator could be in principle modified or replaced by another actuating system, such as a piezoelectric actuator, to have real-time precise and fast displacement control, which would be especially desirable for dynamic tests involving high strain rates or cyclic loads. Application of the present device to single atomic layer material samples will be the aim of future investigations.

\section{Methods}

Sample preparation: Two kinds of aluminum samples, with the shape of microwire with a diameter of $18 \mu \mathrm{m}$ and gage length $\sim 500-800 \mu \mathrm{m}$ and microfilms with thickness of $800 \mathrm{~nm}$, width $\sim 1 \mathrm{~mm}$ and gage length $\sim 500-800 \mu \mathrm{m}$, were prepared for testing according to the following procedure.

The sample, either microwire or thin film, was first deposited onto a $625 \mu \mathrm{m}$ thick Silicon substrate provided with a pre-notch $(\sim 300 \mu \mathrm{m}$ deep $)$ on the back. For the experiments reported in the present paper, we used a common commercial adhesive to fix the sample to the substrate at both sides.

In the case of PMMA, a film with about $3 \times 10 \mathrm{~mm}^{2}$ area and $100 \mathrm{~nm}$ thickness was transferred onto a Si substrate that was provided with a pre-notch on both the top and bottom side. The presence of a the pre-notch just below the nanofilm guarantees complete decoupling between the sample and the substrate over a gage length of about $125 \mu \mathrm{m}$, which could be difficult to provide otherwise. No glue was necessary in this case, as the Van deer Waals forces with Si resulted to securely clamp the sample to the substrate up to its failure.

\section{Acknowledgements}

The authors wish to thank Dr. Maria Giovanna Pastore Carbone and Christos Pavlou for providing the PMMA film tested in the present work. N.M.P. and C. G are supported by the European Commission H2020 under the Graphene Flagship Core 2 No. 785219 (WP14 "Polymer composites"). NMP is supported by the European Commission H2020 also under the Fet Proactive "Neurofibres" No. 732344.

\section{References}

[1] Geim AK and Novoselov KS 2007 Nat. Mater. 6183.

[2] Popov VN 2004 Mater. Sci. Eng. R Rep. 43 (3) 61.

[3] Walker LS, Marotto VR, Rafiee MA, Koratkar N, Corral EL 2011 ACS Nano 5 (4) 3182.

[4] Wang S, Cheng Y, Wang R, Sun J, Gao L 2014 ACS Appl. Mater. Interfaces 6 (9) 6481.

[5] Novoselov KS, Geim AK, Morozov SV, Jiang D, Zhang Y, Dubonos SV, Grigorieva IV, Firsov AA 2004 Science 306666. 
[6] Briseno AL, Mannsfeld SCB, Jenekhe SA, Bao Z, Xia Y 2008 Mater. Today 11 (4) 38.

[7] Kaltenbrunner M et al. 2013 Nature 499458.

[8] Anagnostopoulos G et al. 2016 ACS Appl. Mater. Interfaces 822605.

[9] Hu L, Kim HS, Lee JY, Peumans P, Cui Y 2010 ACS Nano 4 (5) 2955.

[10] Yoo E, Kim J, Hosono E, Zhou H, Kudo T, Honma I 2008 Nano Lett. 8 (8) 2277.

[11] Chena KI, Li BR, Chen YT 2011 Nano Today 6131.

[12] Pantano MF, Espinosa HD, Pagnotta L 2012 J. Mech. Sci. Technol. 26 (2) 545.

[13] Zhu Y and Espinosa HD 2005 PNAS 102 (41) 14503.

[14] Haque M.A. and Saif MTA 2002 Exp. Mech. 42 (1) 123.

[15] Kahn H, Ballarini R, Mullen RL, Heuer AH 1999 Proc. Roy. Soc. A 455 (1990) 3807.

[16] Eppell SJ, Smith BN, Kahn H, Ballarini R 2006 J. Roy. Soc. Interface 3 (6) 117.

[16] Zhang D, Breguet JM, Clavel R, Sivakov V, Christiansen S, Michler J 2010 J. Microelectromech. Syst. 9 (3) 663.

[17] Brown JJ, Suk JW, Singh G, Baca AI, Dikin DA, Ruoff RS, Bright VM 2009 Sens. Actuators A 1551.

[18] Abbas K, Alaie S, Leseman ZC 2012 J. Micromech. Microeng. 22125027.

[19] Pantano MF, Bernal RA, Pagnotta L, Espinosa HD 2015 Meccanica 50 (2) 549.

[20] Pantano MF and Pugno NM 2014 J. Europ. Ceram. Soc. 342767.

[21] Ramachandramoorthy R, Milan M, Lin Z, Trolier-McKinstry S, Corigliano A, Espinosa H 2018 Extreme Mech. Lett. 2014.

[22] Zhang P, Ma L, Fan F, Zeng Z, Peng C, Loya PE, Liu Z, Gong Y, Zhang J, Zhang X, Ajayan PM, Zhu T, Lou J 2014 Nat. Comm. 153782.

[23] Pérez Garza HH, Kievit EW, Schneider GF, Staufer U 2014, Nano Lett. 14 (7) 4107.

[24] Lee C, Wei X, Kysar JW, Hone J 2008 Science 321385.

[25] Lee GH, Cooper RC, An SJ, Lee S, van der Zande A, Petrone N, Hammerberg AG, Lee C, Crawford B, Oliver W, Kysar JW, Hone J 2013 Science 3401073.

[26] Obraztsov AN 2009 Nat. Nanotech. 4212.

[27] Li X et al. 2009 Science $\mathbf{3 2 4} 1312$.

[28] Suk JW, Kitt A, Magnuson CW, Hao Y, Ahmed S, An J, Swan AK, Goldberg BB, Ruoff RS 2011 ACS Nano 5 (9) 6916.

[29] Read DT and Dally JW 1993 J. Mater. Res. 8 (7) 1542.

[30] Sharpe WN Jr, Yuan B, Edwards RL 1997 J. Microelectromech. Syst. 6 (3) 193.

[31] Saif MTA, MacDonald NC1996 Sens. Actuators A 5265.

[32] Chen X, Kirsch BL, Senter R, Tolbert SH, Gupta V 2009 Mech. Mater. 41839. 
[33] Kim JH, Nizami A, Hwangbo Y, Jang B, Lee HJ, Woo CS, Hyun S, Kim TS 2013 Nat. Comm. 42520.

[34] Naraghi M and Chasiotis I 2009 J. Microelectromech. Syst. 18 (5) 1032.

[35] Wang B, Chen X, Pan F, Mao J, Fang Y 2015 Trans. Nonferrous Met. Soc. China 252481.

[36] Yu H, Lu C, Tieu K, Liu X, Sun Y, Yu O, Kong C 2012 Sci. Rep. 2772. 